\title{
The quality of mathematics teaching: a central goal in mathematics teacher education
}

\author{
Despina Potari ${ }^{1}$
}

Published online: 10 November 2017

(C) Springer Science+Business Media B.V., part of Springer Nature 2017

This is my first editorial as editor-in-chief of the Journal of Mathematics Teacher Education. It is my honor to be in this position, and I thank Olive Chapman for trusting me with this responsibility. Olive, as editor-in-chief, has been an inspiring and collaborative person for all of us who have worked with her as associate editors. The current editorial team (me and the four associate editors, Kim Beswick, Gwentolyn Lloyd, Salvador Llinares, and Jeppe Skott) would like to thank Olive for her leadership, which provided the foundation for working with her in moving the journal forward.

With this issue, the JMTE completes 20 years since its first issue was published in 1998. During these years, the JMTE has been a leading journal in mathematics education, reporting high-quality research in mathematics teacher education (see the current review of Williams and Leatham 2017). This research increasingly considers the complexity of mathematics teaching and mathematics teacher education, values the teaching profession, and tries to understand the relation between mathematics, mathematics learning, mathematics teaching, and mathematics teacher education. The number of papers submitted to the JMTE has been continuously increasing, reaching an average of about 160 papers per year during the last decade. The acceptance rate has dropped to (approximately) 15\%, a sign of the demands for maintaining the high quality of the research published in the journal.

In her first editorial as editor-in-chief, Olive discussed what is the field of research on mathematics teacher education as represented in the JMTE (Chapman 2011). She stated that mathematics teacher education is the central focus of the journal, but that studies on mathematics teachers and mathematics teacher educators are also published, as they inform mathematics teacher education research. This continues to be the central scope of the $J M T E$, but is enriched by new theoretical lenses and methodologies allowing better

Despina Potari

dpotari@math.uoa.gr

1 Athens, Greece 
understanding of the processes of mathematics teacher education and the way it frames the development of mathematics teaching and learning, taking into account cultural, institutional, and social contexts.

All the papers in this issue focus on mathematics teacher education practices that can improve the quality of mathematics teaching. Collaborative teacher planning, teaching, and reflection is a main teacher education practice in all the papers. The paper by HallmanThrasher addresses the way in which prospective elementary school teachers elicit and respond to students' thinking in the context of their field experiences. The goal of teacher education is to support prospective teachers to offer students rich mathematical experiences through collaborative lesson planning and enactment of highly demanding problemsolving tasks with pairs of students as well as reflection on this enactment. A similar focus can be seen in the study of Kumar, Subramaniam, and Naik, where a group of practicing middle school teachers collaborated in planning contexts and representations for introducing integers and the operations of addition and subtraction in their classrooms and reflected on these experiences. The study of Lesseig et al. uses similar approaches to mathematics teacher education in the case of leaders' professional development. The study uses video cases showing the interaction between leaders and mathematics teachers as the main avenue for promoting leaders' noticing and reflection. Seventy-two leaders with different roles and responsibilities participated in seminars in repeated cycles that included: working on solving and analyzing mathematical tasks, noticing and interpreting incidents in video cases showing interactions between leaders and mathematics teachers, offering professional development courses to teachers, and reflecting on their experiences.

The theoretical and methodological frameworks used in these papers relate to constructs that have been developed and used in classroom research and/or in studies related to mathematics teachers. The study of Hallman-Thrasher combines enactment of cognitively demanding tasks and the math-talk components (e.g., questioning, explaining mathematical thinking, source of ideas, and responsibilities for learning) to study the quality of mathematics teaching in relation to mathematics teacher education experiences. The definition of data segments with different levels of cognitive demand and their relation to the prospective teachers' actions to elicit and respond to student thinking allowed the author to scrutinize the factors that seem to influence the variation of the cognitive demand and to trace the process of the development of the quality of mathematics teaching. Kumar, Subramaniam, and Naik use the construct of specialized content knowledge in the area of integers and their operations, emphasizing the mathematical dimensions of mathematics learning and teaching. The authors deploy a framework of integer meanings that they developed based on previous research with teachers in a professional development context. The data analysis focuses on the challenges that teachers faced and the process of constructing specialized content knowledge for teaching integers. The study of Lesseig et al. uses theoretical constructs developed in the context of mathematics classroom research (sociomathematical norms, practices for orchestrating productive mathematical discussions) and of work with teachers (specialized content knowledge, professional noticing) in the education of leaders. In the analysis, these constructs were used in combination with grounded theoretical approaches to study what teachers noticed in the video cases, and how.

Hallman-Thrasher found that preparing prospective teachers to anticipate students' responses does not necessarily guarantee high-quality mathematics teaching. Eliciting and responding to students' responses is a rather complex process that is related to the pressure of real-time teaching. However, teacher education that supports prospective teachers to anticipate students' responses and to plan, enact, and reflect on lessons may have a positive 
impact in eliciting and responding to student thinking. The findings of Kumar, Subramaniam, and Naik's study indicate that, when teachers become aware of the meanings of integers and their operations, they: use context-based representations rather than formal models for teaching integer operations, link integer meanings to representations and contexts, and design contexts for teaching that create rich conceptual experiences for the students. The study of Lesseig et al. offers insights into the particular characteristics of professional development seminars and courses that can contribute to leaders' professional learning. They propose the following professional development principles for leaders: defining learning goals for teachers and differentiating teachers' and students' needs, focusing on development of specialized content knowledge, and exemplification of instances from video cases where mathematical goals for teacher learning are articulated.

Closing this editorial, I would like to thank all the previous editors of the JMTE for offering me the opportunity to be a part of the journal and contribute to its development. Tom Cooney invited me as a reviewer, Barbara Jaworski invited me to be a member of the Editorial Board, Peter Sullivan asked me to become an associate editor, and Olive Champman offered me a great challenge and responsibility by inviting me to become editor-in-chief. The whole process has been an important learning experience for me and for my own development as a researcher and mathematics teacher educator. Over these years, I had the opportunity to interact with colleagues from different parts of the world and to better comprehend the role of culture in the development of research, to consider a number of dilemmas related to the quality and relevance of research into mathematics teaching and mathematics teacher education, to face challenges about balancing quality and inclusion, to reflect on my own work as a researcher and mathematics teacher educator, and to try out new ideas and resources that my colleagues around the world have reported in the JMTE. I will continue this journey, and in collaboration with the other members of the editorial team, the members of the Editorial Board, the reviewers, and the colleagues who choose the JMTE as a place to publish their work, will try to continue what has been achieved in these two decades by being open to new directions of research in mathematics teacher education.

\section{References}

Chapman, O. (2011). The field of research in mathematics teacher education. Journal of Mathematics Teacher Education, 14(4), 247-249.

Williams, S. R., \& Leatham, K. R. (2017). Journal quality in mathematics education. Journal for Research in Mathematics Education, 48(4), 369-396. 